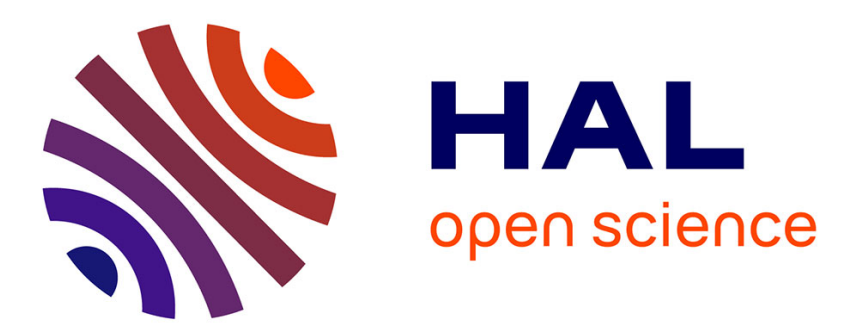

\title{
Le chercheur en humanités digitales : un cas particulier de travailleur du savoir ? \\ Olivier Le Deuff
}

\section{To cite this version:}

Olivier Le Deuff. Le chercheur en humanités digitales : un cas particulier de travailleur du savoir ?. Communication et Management, 2017, 14 (1), pp.55. 10.3917/comma.141.0055 . hal-02480525

\section{HAL Id: hal-02480525 \\ https://hal.science/hal-02480525}

Submitted on 16 Feb 2020

HAL is a multi-disciplinary open access archive for the deposit and dissemination of scientific research documents, whether they are published or not. The documents may come from teaching and research institutions in France or abroad, or from public or private research centers.
L'archive ouverte pluridisciplinaire HAL, est destinée au dépôt et à la diffusion de documents scientifiques de niveau recherche, publiés ou non, émanant des établissements d'enseignement et de recherche français ou étrangers, des laboratoires publics ou privés. 
1. Version pré-print Le Deuff, O (2017) «Le chercheur en humanités digitales: un cas particulier de travailleur du savoir? »Communication management, vol. 14, $\mathrm{n}^{\mathrm{o}} 1,2017, \mathrm{p}$. 55-69.

\section{Le chercheur en humanités digitales : un cas particulier de travailleur du savoir?}

Les humanités digitales constituent une rénovation des sciences humaines et sociales sur les plans méthodologiques, épistémologiques et pédagogiques (Svensson, 2010). Le mouvement est porteur d'une remise en cause des processus classiques de la recherche et de l'enseignement. C'est par exemple le cas des ThatCamp ${ }^{l}$ qui proposent une manière différente de concevoir les colloques en incitant tous les inscrits à réellement participer (Thély, Serres, Le Deuff, 2014).

Les humanités numériques ou digitales ${ }^{2}$ modifient les méthodes de travail des chercheurs impliqués dans des projets qui mobilisent des dispositifs numériques. Les changements concernent donc autant le chercheur lui-même que les éléments organisationnels. Nous souhaitons examiner ici les chercheurs en humanités digitales comme un cas particulier de travailleurs du savoir en montrant l'évolution de leurs méthodes et stratégies de gestion des connaissances. Cette étude s'appuie notamment sur une enquête bilingue (anglais, français) réalisée en 2013 auprès d'une centaine de chercheurs $(n=106)$ impliqués dans les humanités digitales $^{3}$. Ces chercheurs étaient majoritairement des initiés- au sens d'insiders, c'est-à-dire des personnes investies dans ces domaines. Nous entendons par chercheur en Humanités Digitales, un chercheur dans le domaine des sciences humaines et sociales qui utilise des méthodes et outils de type informatique ou numérique pour améliorer ses analyses de données, ses traitements de corpus, ses méthodes d'organisation, de gestion de l'information ainsi que ses stratégies de communication et de publication. Les chercheurs ayant répondu à l'enquête occupent différents types de postes, en tant que professeurs titulaires, mais également comme ingénieurs de recherche titulaires ou contractuels ${ }^{4}$.

Nous souhaitons montrer ici que les changements qui s'opèrent, se déroulent d'abord à l'échelon individuel avant d'impacter les niveaux organisationnels et institutionnels. Cette étude s'inscrit ainsi dans le champ des sciences de l'information et de la communication en prenant en compte les aspects informationnels, communicationnels, sociotechniques et

\footnotetext{
1 The Humanities and Technology Camp sont des conférences qui cherchent à rénover les modes de communication et d'interactions Durant les manifestations scientifiques.

${ }^{2}$ On ne discutera pas de la traduction adéquate dans cet article. Nous avons cependant fait le choix de la traduction par humanités digitales.

${ }^{3}$ Il s'agit des résultats de recherche du projet Humanlit (Humanités numériques et littératies) soutenu par l'ISCC (Institut de la Communication du CNRS. Les résultats sont disponibles et librement réutilisables ici : http://humanlit.hypotheses.org/206

4. Sur les aspects de la diversité des digital humanities, voir l'étude de Marin Dacos. La stratégie du Sauna finlandais, Juin 2013. Le blog d'Homo-numericus <http://bn.hypotheses.org/11138>
} 
organisationnels de la gestion des connaissances (Béguin-Verbrugge, 2008). Nous prenons ici l'exemple d'enseignants-chercheurs car ils sont depuis longtemps dans une logique d'échanges en dehors de leur institution première depuis les réseaux de correspondance comme celui de Marin Mersenne jusqu'aux divers collèges invisibles. Le numérique ne fait que faciliter ces possibilités d'échanges de façon plus ou moins informelle avec des réseaux spécialisés au niveau de la recherche qui tentent de faciliter les mises en relation et le partage de documents comme ResearchGate et Academia.edu. Toutefois ces plateformes sont dans l'esprit des entreprises issues du mouvement 2.0 en cherchant à monétiser les données accumulées. Les chercheurs en humanités digitales correspondent assez bien à la description des chercheurs 2.0 (Gallezot, Le Deuff, 2009) en présentant des pratiques numériques avérées à plusieurs niveaux de leur travail: recherche d'informations et d'articles, collecte de données, outils de traitement de données, partage et échange d'informations sur les réseaux ${ }^{5}$. Ceux qui sont investis dans les humanités digitales ont d'ailleurs une présence affirmée sur twitter $^{6}$, qui est considéré comme le réseau de base pour tout chercheur dans les humanités digitales. Dan Cohen, directeur de la bibliothèque numérique américaine et pionnier des humanités digitales, recense 353 personnes dans une liste ${ }^{7}$ qui regroupe les principaux chercheurs impliqués, tandis que Martin Grandjean, doctorant en histoire à l'Université de Lausanne en recense près de $1000^{8}$. Qu'on soit dans une application restrictive ou au contraire élargie des chercheurs en humanités digitales, nous souhaitons montrer ici les spécificités de ce public et en quoi ils peuvent constituer un modèle intéressant d'organisation des connaissances renouvelé à tous les niveaux (individuel et collectif, au niveau des découpages disciplinaires et des recompositions institutionnelles, etc.)

\section{Le chercheur en Humanités digitales : un cas particulier de travailleur du savoir}

Les chercheurs et notamment les enseignants-chercheurs en sciences humaines et sociales constituent un public particulier de travailleurs du savoir. Nous faisons le choix ici d'étudier un public restreint de travailleurs de savoirs parmi les universitaires : ceux qui sont investis dans les humanités digitales. Nous considérons comme chercheurs en humanités digitales les chercheurs dont les travaux reposent sur des outils numériques et de façon plus générale, tous ceux qui manifestent de l'intérêt pour cette communauté notamment en participant à des manifestations de type ThatCamp. Ce sont donc des travailleurs du savoir qui en couvrent les différents aspects car ce sont également des créateurs de savoir. En effet, Davenport considère les travailleurs du savoir comme des «personnes avec un haut degré d'éducation ou d'expertise, dont le premier objectif de leur activité consiste à créer, distribuer ou appliquer

\footnotetext{
${ }^{5}$ Notre enquête montre que $62,26 \%$ des répondants utilisent les flux rss par exemple et qu'ils sont près de 67 $\%$ à utiliser des moteurs de recherche spécialisés.

${ }^{6} 67,92 \%$ sont présents sur Twitter. Toutefois, l'enquête a également été diffusée via le réseau twitter ce qui peut expliquer ce résultat.

${ }^{7} \mathrm{https} / / /$ twitter.com/dancohen/lists/digitalhumanities/members

${ }^{8} \mathrm{https}: / /$ twitter.com/GrandjeanMartin/lists/digital-humanities
} 
la connaissance (...) Il y a différents types de travailleurs du savoir, l'un applique la connaissance et l'autre la crée »".

\subsection{Un producteur de savoirs}

La distinction entre ceux qui utilisent l'information et ceux qui la créent est fréquemment faite pour distinguer les différents types de travailleurs du savoir. Cette distinction devient parfois difficile notamment avec les dernières évolutions du numérique depuis le phénomène web 2.0 où l'usager devient également créateur et diffuseur d'information et de données. Dès lors, il paraît difficile d'opérer une stricte distinction entre des créateurs et des exécutants et ceux que Jean Pierre Bouchez (Bouchez, 2004) nomme des professionnels du savoir. Alan Liu rassemble sous le vocabulaire de travailleurs du savoir des métiers différents qu'il résume dans l'équation suivante (Liu, 2004a, p. 392) :

Travailleurs du savoir $=$ universitaires + intelligentsia à la fois technique, professionnelle et managériale de la diversité des employés de bureau. ${ }^{10}$

Le concept de travailleur du savoir vient des travaux de Peter Drucker qui a développé le concept dans une vision de type managérial. Nous nous intéressons ici principalement à son application dans le domaine scientifique dans une approche qui s'inscrit dans la lignée des outils d'organisation de l'information et d'amélioration de la recherche. Nous songeons ainsi aux travaux précurseurs de Paul Otlet, d'Emmanuel Goldberg, de Vannevar Bush, dee Vasily Nalimov et de Derek John de Solla Price (scientométrie).

Le travailleur du savoir des humanités digitales est un travailleur intellectuel (Guitton, 1951), non pas en tant qu'intellectuel public (Ory, Sirinelli, 2004) - même si ce n'est pas incompatible -, mais comme personne qui use de ses facultés intellectuelles dans son travail quotidien. Dans le cas du chercheur en humanités digitales, il convient de le considérer à la fois comme un consommateur et comme un producteur d'informations qui cherche à optimiser ses méthodes de recherche d'informations, de traitement de ses données et de communication scientifique. Sa relation avec la technique apparaît ici essentielle tant les dispositifs du plus simple au plus complexe participent de son activité.

\subsection{Le renouvellement des modes de production de savoirs}

En effet, la production des connaissances apparaît essentielle, mais elle marque une évolution dans les formes qu'elle peut prendre comme le montre notre enquête (tableau $1)$.

\begin{tabular}{|c|l|l|}
\hline Réponse & Décompte & Pourcentage \\
\hline Les banques et / ou bases & 64 & $60.38 \%$ \\
\hline
\end{tabular}

\footnotetext{
${ }^{9}$ Interview pour la revue Ubiquity : http://www.acm.org/ubiquity/interviews/v6i34_davenport.html

${ }^{10}$ Citation originale $:$ « Knowledge workers $=$ academic intellectuals $+($ technical + professional + managerial $)$ inteligentsia $=$ trailing edge of clerical workers.
} 


\begin{tabular}{|c|r|r|}
\hline \multicolumn{2}{|l|}{ de données production de } \\
\hline $\begin{array}{c}\text { La de } \\
\text { documents d'analyse de } \\
\text { résultats }\end{array}$ & 62 & $58.49 \%$ \\
\hline $\begin{array}{c}\text { La visualisation de } \\
\text { résultats et / ou de données }\end{array}$ & 62 & $58.49 \%$ \\
\hline Les livres électroniques & 57 & $53.77 \%$ \\
\hline $\begin{array}{c}\text { Les blogs ou carnets de } \\
\text { recherche }\end{array}$ & 53 & $50.00 \%$ \\
\hline
\end{tabular}




\section{Tableau 1. Quelles nouvelles formes d'écriture et de productions scientifiques mériteraient d'être mieux valorisées et évaluées ? $^{11}$}

Cette variété dans la production implique également un enjeu autour de la diffusion de la connaissance notamment autour des réflexions en ce qui concerne les différentes formes du libre accès. Par conséquent, un nouveau modèle des savoirs et de la connaissance est en train de se réaliser dans les humanités digitales. Il tend à se différencier du modèle classique qui repose sur des processus de sélection et de contrôle par les pairs avec la délivrance des diplômes et les autorisations à publier en élargissant les processus de publication scientifique et en accroissant l'importance de l'évaluation a posteriori par les mécanismes de citations y compris sur les réseaux sociaux, lieux des métries alternatives ce qui suppose une évolution des modèles économiques de la production scientifique. En effet, la logique prédominante qui repose sur la publication dans des revues reconnues afin de garantir une meilleure reconnaissance des pairs et l'inscription dans des logiques de citations afin d'obtenir un index favorable apparaît trop circonscrite au modèle de l'article issu de l'imprimé. Cette économie de la publication favorise les revues payantes et les éditeurs privés, mais ne permet pas d'exprimer pleinement les possibilités des environnements numériques. La nouvelle économie des connaissances des humanités digitales implique un renouveau dans le type de publication et une délivrance au sens où l'entendent Claire Clivaz et Dominique Vinck (Clivaz et Vinck, 2014), c'est-à-dire une sortie de la logique figée issue de la culture imprimée et du livre, pour aller sur des modèles davantage ouverts. La meilleure piste serait alors de redéfinir l'article sous la forme d'une Application Programming Interface (API) (Le Deuff, 2014).

Le modèle économique est encore difficile à saisir d'autant que de nouveaux acteurs tentent d'en saisir les bénéfices tels les réseaux sociaux, ResearchGate ou Academia.edu. Quoi qu'il en soit, les modèles vont demeurer très nettement dans la logique de la documentalité (Ferraris, 2012) tant l'activité de recherche requiert l'inscription et l'enregistrement dans des formes plus ou moins complexes. Quelle que soit la forme de production choisie au final, la production d'une forme documentaire constitue le témoignage de l'activité de recherche. Ce sont ses formes, ses modes d'évaluation et de valorisation qui sont amenés à évoluer dans les années qui viennent, non sans heurts probablement comme en témoignent les inquiétudes autour de l'Open Access et les revendications financières des éditeurs privés et des plateformes comme Cairn. L' économie de la connaissance implique donc des réflexions en ce qui concerne la chaîne de productions de valeurs et ses différents types d'évaluations qui ne peuvent demeurer sur la seule question du financement des travaux de recherche mais sur les compétences à développer pour faciliter l'émergence de nouvelles formes de productions.

\subsection{De nouveaux besoins de formations}

Les évolutions du type de production impliquent de nouvelles compétences qui peuvent s'échanger au sein des communautés de pratique. Il est encore difficile de pouvoir distinguer des formes pleinement stabilisées de systèmes de gestion de connaissances propres aux humanités digitales car il n'y a pas véritablement de bases de connaissances type KM. Quelques projets ont tenté de rassembler des éléments de connaissances sous forme d'annuaire, comme le projet Bamboo ${ }^{12}$ (Dombrowski, 2014) qui est désormais arrêté, tandis que le projet Programminghistorian ${ }^{13}$ devient davantage l'affaire de spécialistes avec beaucoup de tutoriaux. La logique actuelle devient davantage participative en incluant plus tôt les étudiants dans les projets et donc dans la création de connaissances, mais également en

\footnotetext{
${ }^{11}$ Plusieurs réponses étaient possibles.

${ }^{12} \mathrm{http}: / / \mathrm{www}$.projectbamboo.org/

${ }^{13} \mathrm{http}$ ///programminghistorian.org/
} 
procédant de façon plus transdisciplinaire ce qui peut bouleverser l'organisation des connaissances, notamment au niveau des découpages disciplinaires. Cela pose d'ailleurs plusieurs difficultés en matière de recrutement pour un chercheur dont les compétences ne sont pas totalement rattachées à un domaine disciplinaire plus classique. Au niveau institutionnel, les centres d'humanités digitales bouleversent également le jeu classique des laboratoires et des départements organisés initialement dans la logique des disciplines. De ce fait, les humanités digitales impliquent une réorganisation des connaissances qui passent par des alliances disciplinaires qui facilitent l'interdisciplinarité, mais qui reposent en fait sur des logiques de transdisciplinarité, ce que beaucoup d'auteurs soulignent (Hayles, 2012), d'autant qu'il s'agit de faire circuler savoirs et savoir-faire d'une discipline à l'autre. D'ailleurs, le manifeste du ThatCamp à Paris en 2010 soulignait ce caractère transdiciplinaire :

\footnotetext{
1. Le tournant numérique pris par la société modifie et interroge les conditions de production et de diffusion des savoirs.

2. Pour nous, les digital humanities concernent l'ensemble des Sciences humaines et sociales, des Arts et des Lettres. Les digital humanities ne font pas table rase du passé. Elles s'appuient, au contraire, sur l'ensemble des paradigmes, savoir-faire et connaissances propres à ces disciplines, tout en mobilisant les outils et les perspectives singulières du champ du numérique.

3. Les digital humanities désignent une transdiscipline, porteuse des méthodes, des dispositifs et des perspectives heuristiques liés au numérique dans le domaine des Sciences humaines et sociales
}

Les humanités digitales sont donc un lieu de rassemblement et de mise en commun. Peu considèrent d'ailleurs qu'il faille faire des humanités digitales une discipline autonome ${ }^{14}$. La gestion des connaissances doit être également ici comprise dans ses deux acceptions, à la fois en tant que savoir, mais aussi en tant que relations interpersonnelles. C'est d'autant plus important que pour le chercheur en humanités digitales, il devient de plus en difficile de se penser en tant que chercheur isolé et qu'il lui faut mobiliser différentes techniques dont il n'a pas toujours la totale maîtrise, d'où l'intérêt de connaître celui ou celle qui pourra soit l'aider dans son travail, soit réaliser la mission de manière plus efficiente. Le fait que les projets requièrent un plus grand nombre de personnes avec des profils différents entraînent une meilleure capacité à collaborer avec des métiers comme les ingénieurs, les archivistes et les bibliothécaires (Pouyllau, 2014). Toutefois, il apparaît que la question des besoins de formation en matière de gestion de projet apparaît moins opportune que la formation à des aspects davantage techniques et pratiques (tableau 2). On note toutefois des disparités assez fortes sur cette question en fonction du genre. Les chercheures ayant répondu se montrent plus intéressées $(55,56 \%)$ par ce type de formation que leurs homologues masculins $(41,17 \%)$.

\begin{tabular}{|l|l|l|}
\hline Réponse & Décompte & Pourcentage \\
\hline $\begin{array}{l}\text { Formation pratique à } \\
\text { 1'utilisation d'outils } \\
\text { spécifiques }\end{array}$ & 84 & $79.25 \%$ \\
\hline $\begin{array}{l}\text { Formation } \\
\text { méthodologique }\end{array}$ & 79 & $74.53 \%$ \\
\hline
\end{tabular}

\footnotetext{
14 Plus de $57 \%$ des répondants répondent par la négative à la question suivante : Pensez-vous que les humanités numériques doivent être considérées comme une discipline à part entière ?
} 


\begin{tabular}{|l|r|r|}
\hline Formation théorique & 66 & $62.26 \%$ \\
\hline Gestion de projet & 50 & $47.17 \%$ \\
\hline Autre & 16 & $15.09 \%$ \\
\hline
\end{tabular}

Tableau 2. Quel type de formation serait nécessaire pour les chercheurs?

Le besoin de se former en agrégeant de nouvelles compétences tout au long de la carrière apparaît comme une nécessité intrinsèque à la profession de chercheur. Cependant, cette gestion des connaissances implique du temps. Chez les chercheurs en humanités digitales, il ne s'agit pas de se placer en position de délégation en confiant à des ingénieurs plus à l'aise le soin de réaliser l'ensemble des opérations techniques, mais bien de pouvoir effectuer ce travail soi-même ou si besoin en étroite coopération. Ce besoin à la fois de se former et d'expérimenter va au-delà de la division traditionnelle entre le travail et les loisirs. Difficile de distinguer ce qui relève du professionnel et de l'intérêt privé, si bien que les temps s'en trouvent peu aisés à découpler parfois. Les travailleurs du savoir qui utilisent fréquemment les outils numériques, savent qu'il devient peu aisé également d'opérer cette distinction entre travail et loisirs du fait que les activités se mêlent et s'effectuent bien souvent sur un même dispositif de type numérique.

\section{Un nouveau paradigme informationnel}

Le numérique est porteur d'éléments nouveaux en matière de changements organisationnels et de manière de travailler au point d'ailleurs que chez certains travailleurs du savoir la frontière entre ce qui relève du professionnel et du loisir n'est pas toujours nette. C'est particulièrement le cas chez les chercheurs et notamment ceux impliqués dans des projets numériques.

\subsection{L'influence des lois du «cool »?}

Faut-il y voir la logique des «lois du cool $»^{15}$, cette volonté de faire paraître le travail plus agréable et de même de l'affirmer haut et fort, qu'avait déjà mis en avant Alan Liu (Liu, 2004), professeur à l'Université de Californie de Santa Barbara et théoricien critique des Digital Humanities? Ce dernier avait remarqué que la vision majoritaire des travailleurs du savoir était essentiellement d'ordre managérial et orientée valorisation économique et qu'elle présentait des différences avec la logique des chercheurs et enseignants-chercheurs dont les savoirs et leurs transmissions ne reposent pas nécessairement sur une marchandisation immédiate. Pour autant, Alan Liu avait pu observer que les chercheurs et enseignants en sciences humaines et sociales adhéraient de plus en plus à certaines visions managériales des travailleurs du savoir, ce que Readings (Readings, 1996) avait contribué à dénoncer en décrivant une pseudo culture de l'excellence ou d'une inculture du chiffre comme le décrit Yves Citton (Citton, 2015). Cette culture du cool, alors qu'elle prétend jouer sur la diversité accroît l'uniformité culturelle en produisant une doxa managériale niant la division travail et

\footnotetext{
${ }_{15}$ Le concept des «lois du cool» est décrit par Liu comme un mécanisme qui conduit à privilégier l'information à la connaissance, l'immédiat à la réflexion. Liu montrait que l'université n'avait pas su élaborer une critique de l'approche managériale et encore moins une critique face à l'emploi du mot «cool» utilisé justement pour éviter toute analyse fine des évolutions du marché du travail.
} 
loisir notamment en rendant le travail davantage attractif. En effet, la logique du cool s'avère une éthique de l'information voire une esthétique de l'information tant il s'agit à la fois de se prétendre cool et de le démontrer notamment par des interfaces dédiées à cet objectif (Liu, 2004b). Du coup, le cool devient une forme d'Ego de l'information, un alter ego qui se révèle parfois en opposition avec la logique info-documentaire de la qualité du contenu pour privilégier une attitude. De plus, le cool réside aussi dans un formalisme, un formalisme dominé par le design, de moins en moins par modelage littéraire et intellectuel, mais davantage graphique et marketing. Un design basé sur une littératie visuelle qui recherche au maximum une clarté qui vise la compréhension des messages visuels (Dondis, 1974). Il se produit alors une recherche esthétique des travailleurs du savoir qui va au-delà des aspects vestimentaires pour s'observer sur le web comme espace de distinction. Le travailleur du savoir peut désormais satisfaire sa recherche esthétique non seulement en termes de consommation, mais également dans une logique de production grâce au web. L'esthétique concerne donc la représentation des connaissances avec des logiques nouvelles basées sur des formes visuelles renouvelées qui consistent à tenter de représenter des interactions observées sur des corpus bien plus grands qui requièrent une lecture à distance (Moretti, 2013).

\subsection{Les nouvelles lectures et accès à la connaissance.}

Les expériences de Lev Manovich (Manovich, 2013) sont souvent citées dans ce cadre. Cela implique par conséquent de nouveaux modes d'accès aux connaissances qui diffèrent de la seule logique de la lecture proche ou exhaustive pour utiliser la médiation d'outils de traitement automatisé pour appréhender des centaines voire des milliers de textes différents. Dès lors, les travailleurs du savoir impliqués dans les humanités digitales recherchent d'autres modes d'accès la connaissance en tentant d'opérer des lectures profondes au sein de corpus de plus en plus grands (fig1.).

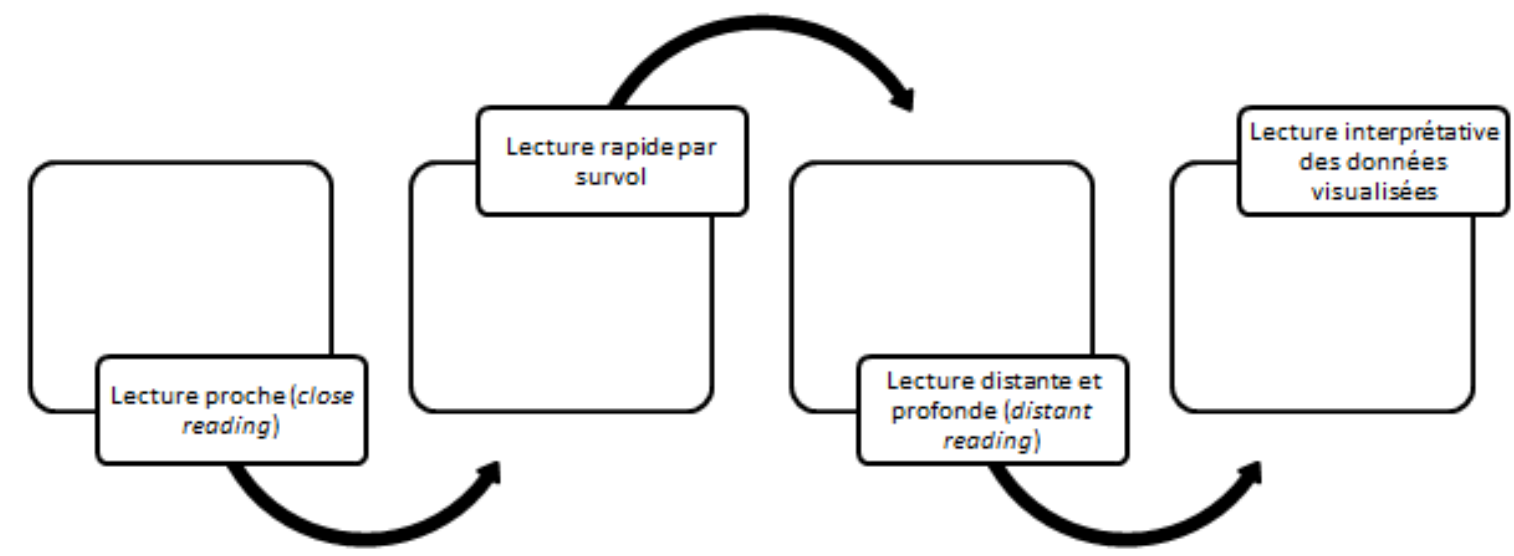

Fig. 1. Les différents types de lecture dans les humanités digitales

Le close reading et le distant reading se mêlent. La médiation numérique (Boustany, Broudoux, Chartron, 2014) poursuit donc sa présence au-delà des logiques de catalogues de 
bibliothèques et de base de données pour retrouver l'information, en devenant présente dans la lecture même des documents et des données et en accompagnant de plus en plus son analyse même. Le savoir se trouve donc de plus en plus digitalisé pour être rendu plus aisément manipulable notamment par des traitements informatiques comme les algorithmes. Seulement, le mouvement des digital humanities cherche désormais à développer en parallèle des potentialités offertes par le traitement numérique et informatique, une nouvelle perspective davantage interprétative et critique voire émotionnelle pour reprendre les propos $\mathrm{du}$ digital humanities manifesto $2.0^{16}$.

\subsection{La dimension critique}

Cette capacité critique est aussi selon Presner (Presner, 2010) une ouverture vers d'autres modes d'organisations des savoirs davantage ancrés dans le numérique, et donc plus éloignés, voire en rupture avec les formes issues de la culture imprimée. C'est l'enjeu d'une dimension critique et analytique des humanités digitales défendues notamment par Alan Liu et David Berry (Berry, 2012) face à une tendance qui prône la fin de la science du fait de la possibilité d'obtenir des données et des visualisations en temps réel. Ce courant provocateur évoquait dans Wired Magazine l'émergence de nouvelles méthodes qui permettraient de décrire la réalité en temps réel. Ce phantasme du big data (Boyd, 2011) comme réponse à tout constituerait le summum des lois du cool, avec une transformation des relations et de la moindre manifestation en données interprétables. Les chercheurs en sciences humaines et sociales et plus particulièrement les chercheurs en humanités digitales, qui manipulent de plus en plus de données, défendent l'importance des disciplines de l'interprétation. Par conséquent, l'utilisation de méthodes et d'outils numériques ne fait pas disparaître la dimension analytique bien au contraire, car cette analyse critique va porter autant sur les résultats que sur les méthodes et les processus pour les obtenir.

Du coup, l'intérêt d'étudier les chercheurs en humanités digitales est clef car ils constituent un bon exemple de travailleurs du savoir qui développent une culture numérique qui exploite davantage le numérique pour améliorer leurs méthodes d'accès à la connaissance face à une surabondance informationnelle. Mais ils doivent également développer de nouvelles capacités à produire une critique des résultats et des méthodes tant il s'agit de concevoir une science renouvelée dans ses méthodes et objets, mais aussi dans son épistémologie et surtout dans sa portée au niveau de la société. L'impact de la transdiscipline se situe alors dans ces missions d'enseignement, sa transmission vers un public étudiant, mais aussi vers un public d'amateur éclairé.

Si ce public spécialisé peut apparaître comme initié, il est probable que de nombreux outils et méthodes vont finir par se diffuser et se démocratiser chez un plus grand nombre de travailleurs du savoir. Mais cette démocratisation passe par un apprentissage des littératies requises et non seulement par une mise à disposition des outils. L'accès à la connaissance

\footnotetext{
${ }^{16}$ A Digital Humanities Manifesto. The Digital Humanities Manifesto 2.0. 29, mai 2009 http://manifesto.humanities.ucla.edu/2009/05/29/the-digital-humanities-manifesto-20/
} 
s'opère de façon différente, tout comme le moyen d'exprimer et de diffuser cette connaissance nouvelle.

\section{Un nouveau mode d'organisation des connaissances pour de nouveaux travailleurs du savoir}

Le modèle des travailleurs du savoir est davantage issu désormais de la sphère commerciale et marketing. Par conséquent l'émergence des digital humanities doit permettre d'offrir une autre vision selon Liu (Liu, 2004 et $2009^{\circ}$ ). Selon Berry, la troisième vague des humanités digitales doit désormais étudier plus largement comment le numérique et notamment le code finit par pénétrer également les modes de travail des humanités.

\subsection{Un changement épistémologique?}

Une des pistes serait de revenir à la lignée du travail intellectuel (Guitton, 1951) et de se placer dans la généalogie des outils de la pensée. L'interrogation porte d'ailleurs sur la définition même de la culture après qu'elle ait été « logicielisée ${ }^{17}{ }^{1}$ ? (Berry, 2012 et 2015). D'où la question de comprendre si le savoir finit par être transformé en information voire simplement en données avec les logiciels. La majorité des répondants à notre enquête considère que les humanités opèrent un changement épistémologique majeur ; reste à savoir lequel précisément.

\begin{tabular}{|l|r|r|}
\hline Réponse & Décompte & Pourcentage \\
\hline 0 pas du tout & 6 & $5.66 \%$ \\
\hline $\mathbf{1}$ & 3 & $2.83 \%$ \\
\hline $\mathbf{2}$ & 8 & $7.55 \%$ \\
\hline $\mathbf{3}$ & 32 & $30.19 \%$ \\
\hline $\mathbf{4}$ & 28 & $26.42 \%$ \\
\hline $\mathbf{5}$ tout à fait & 29 & $27.36 \%$ \\
\hline Sans réponse & 0 & $0.00 \%$ \\
\hline Non affiché & 0 & $0.00 \%$ \\
\hline Total & 106 & $100 \%$ \\
\hline
\end{tabular}

Tableau 3. Pensez-vous que les DH engagent une évolution épistémologique?

Cette évolution épistémologique entrainée par le numérique interroge les fondements universitaires basés sur l'exercice de la raison selon Kant. Il reste néanmoins que la raison, ratio en latin présente un double sens. Celui de l'exercice de la raison, mais aussi celui de calcul et donc du rendu calculable. Il faut donc vraisemblablement comprendre qu'il s'agit d'une relation étroite entre le fait de produire des données interprétables par des traitements

\footnotetext{
17 «Si l'on veut mieux comprendre le numérique, un premier pas pourrait être de problématiser la computationnalité, afin de développer une pensée critique sur la façon dont la connaissance au XXI ${ }^{\mathrm{e}}$ siècle se trouve transformée en information à travers des techniques computationnelles, en particulier au sein des logiciels. Il faut prêter attention à une coïncidence historique frappante entre le fait que l'idée de l'université se trouve actuellement repensée et renégociée et le fait que les technologies numériques soient en train de transformer notre capacité à utiliser et comprendre des informations en dehors des structures traditionnelles du savoir, comme l'université » (Berry, 2015)
} 
logiciels et la capacité à réaliser des interprétations «savantes ». L'université dans la lignée de Humboldt constitue un lieu de rassemblement des savoirs qui conçoit la connaissance davantage comme un processus de formation (Bildung, en tant que formation de l'esprit) plutôt qu'un produit. Toutefois, les sphères universitaires sont des lieux de production de savoir comme autant de preuves et de volontés d'accroître la somme des savoirs disponibles. $\mathrm{Si}$ initialement le processus exige des procédures de contrôle afin de procéder à une vérification de la qualité, notamment des articles scientifiques, les possibilités de diffusion via le numérique changent les logiques issues de la chaîne documentaire ou du circuit du livre pour aller vers des circuits plus courts avec de la communication scientifique davantage directe symbolisés notamment par les carnets ou blogs de recherche ainsi que la diffusion des pre-prints. Dès lors, la logique est clairement celle de l'échange d'information, de données et de connaissances au sein des communautés disciplinaires, mais aussi au-delà. Pour le chercheur, cela implique une inscription au sein de plusieurs cercles, notamment une présence parmi une ou plusieurs communautés de pratiques (Wenger, 1998). Pour les chercheurs en humanités digitales, cette inscription est hybride car elle oblige à conserver quelque peu les réseaux disciplinaires traditionnels tout en développant de nouveaux lieux de présence et d'échange. Outre les listes de diffusion ${ }^{18}$, lieux d'échanges classiques pour constituer des communautés de pratiques, le chercheur en humanités digitales va être également présent sur les réseaux sociaux et plus particulièrement sur twitter qui est devenu le lieu quasi obligé pour les chercheurs investis dans les humanités digitales. Impossible d'imaginer un ThatCamp sans son hashtag dédié par exemple. Twitter illustre parfaitement l'enjeu d'une gestion des connaissances plus complexes et qui mêlent les deux types de connaissances (savoirs + personnes) et qui montrent aussi les frontières de plus en plus floues entre travail et loisir, entre diffusion d'information et expression d'opinion, entre construction d'une e-réputation et valorisation de ses travaux.

\subsection{Une nouvelle économie de la connaissance}

Indéniablement, cette logique de circulation au sein d'espaces différents (sphère scientifique, sphère institutionnelle, communautés de pratiques, réseaux sociaux, etc.) ne peut être comprise d'une façon équivalente à une gestion de connaissances telle qu'elle pourrait se pratiquer en entreprises avec des procédures normalisées qui visent à rendre les connaissances tangibles et consultables, mais dans un cadre contrôlé et souvent fermé. Cette logique ne peut convenir aux chercheurs en humanités digitales qui ont au contraire intérêt à la plus large diffusion possible, si bien que les temps d'édition dans le domaine des sciences sociales et humaines rentrent en conflit avec les logiques sociales de l'immédiateté. Cette logique maximale de diffusion implique également une capacité de veille, de repérage d'informations, de stockage et de traitement personnel de plus en plus élaborée pour filtrer les éléments qui pourront servir au chercheur ultérieurement. Dès lors, la gestion des connaissances du chercheur en humanités digitales s'étend du PKM (Pollard, 2008) au Massive Km (fig.2).

\footnotetext{
${ }^{18}$ La liste Humanist, créée par Willard McCarthy est ainsi un lieu incontournable des digital humanities d'autant qu'elle fut conçue à l'époque où on parlait davantage d'humanities computing. Dans le registre francophone, c'est la liste DH qui prend de l'ampleur. On notera le paradoxe que la liste de diffusion francophone a fait le choix de la non-traduction de l'expression anglophone qui se trouve ici réduite à un sigle.
} 
- Le PKM ou Personal Knowledge Management se développe par les facilités d'accès à l'information et au savoir - qui scit ubi scientia sit, ille est proximus habenti ${ }^{19}$ - comme le rappelait en exergue du traité de documentation, Paul Otlet. Le pkm implique une organisation personnelle efficace qui mêle un grand nombre d'outils différents dont la plupart sont situés sur une même machine et qui sont utilisables via des dispositifs en ligne ou hébergés sur des clouds. Plus le dispositif de PKM est optimisé, plus les possibilités de traitement de données et d'informations permettent une production de nouvelles connaissances qui peuvent être plus aisément partagées. L'enjeu pour le chercheur en humanités digitales est de pouvoir passer d'une application à l'autre pour faire transiter les informations et données recueillies. C'est le stade essentiel pour envisager une évolution de la gestion des connaissances et un partage amélioré en prenant conscience que la collaboration (Deegan, 2012) soit dans une logique gagnantgagnant. Ce stade essentiel rentre parfois en contradiction avec les intérêts de l'organisation et de l'entreprise, car il confère une forme d'indépendance même si cela peut générer un surplus de travail (Le Deuff, 2012º).

- L'OKM (Organization Knowledge Management) se situe au niveau de l'organisation, c'est le niveau le plus classique de la gestion des connaissances depuis les travaux de Nonaka et Takeuchi. En ce qui concerne, le chercheur en humanités digitales, cela concerne plutôt son université ou son laboratoire. À ce stade, la gestion des connaissances s'appuie sur le partage de savoirs entre collègues de façon informelle ou plus institutionnelle (séminaires, etc.) et concerne aussi toutes les étapes de transmission de savoirs envers les étudiants et les doctorants. La transmission ne devant pas être d'ailleurs uniquement à sens unique du professeur vers l'étudiant, notamment dans le cadre de participation à des projets qui laissent la possibilité aux étudiants de pouvoir également transmettre des informations, des réalisations, des découvertes à leur professeur et à leurs camarades. D'autres pistes peuvent être évoquées, notamment celles qui consistent justement à créer des lieux qui facilitent la transdicipline avec des départements orientés humanités digitales ou des centres en humanités digitales, conçus comme des laboratoires numériques où on mutualise les outils et les compétences avec parfois des postes dédiés. Certaines initiatives se déroulent d'ailleurs au sein même des bibliothèques universitaires. (Posner, 2013, Novwiskie, 2013)

- Le CKM, ou le Community Knowledge Management, se situe à un niveau supérieur, fréquemment assimilable aux communautés de pratique. Le chercheur en humanités digitales va échanger ici au sein de ses communautés d'intérêts. L'articulation avec le PKM est extrêmement forte et aisée via le numérique. Cela concerne les relations avec ses communautés disciplinaires, mais également les communautés limitrophes et la communauté transdisciplinaire des humanités digitales.

- Le massive Km ou hyper KM est le stade de la diffusion à un public élargi, notamment vis-à-vis d'autres chercheurs, mais aussi à destination d'amateurs éclairés ou de publics de professionnels. L'objectif étant la diffusion élargie de l'information et de la connaissance notamment sous des formes documentaires accessibles. C'est l'enjeu des

\footnotetext{
${ }^{19}$ Que celui qui sait où se trouve le savoir soit tout près de le posséder
} 
humanités digitales populaires qui permettent à tous de devenir des humanistes digitaux (Little, 2011). Évidemment, il est aisé de songer d'emblée à des dispositifs type MOOC (Massive Online Open Course) dont l'objectif est de toucher un public beaucoup plus important qu'à l'accoutumée, mais cela peut prendre des formes moins élaborées avec simplement la mise à disposition de documents librement consultables.

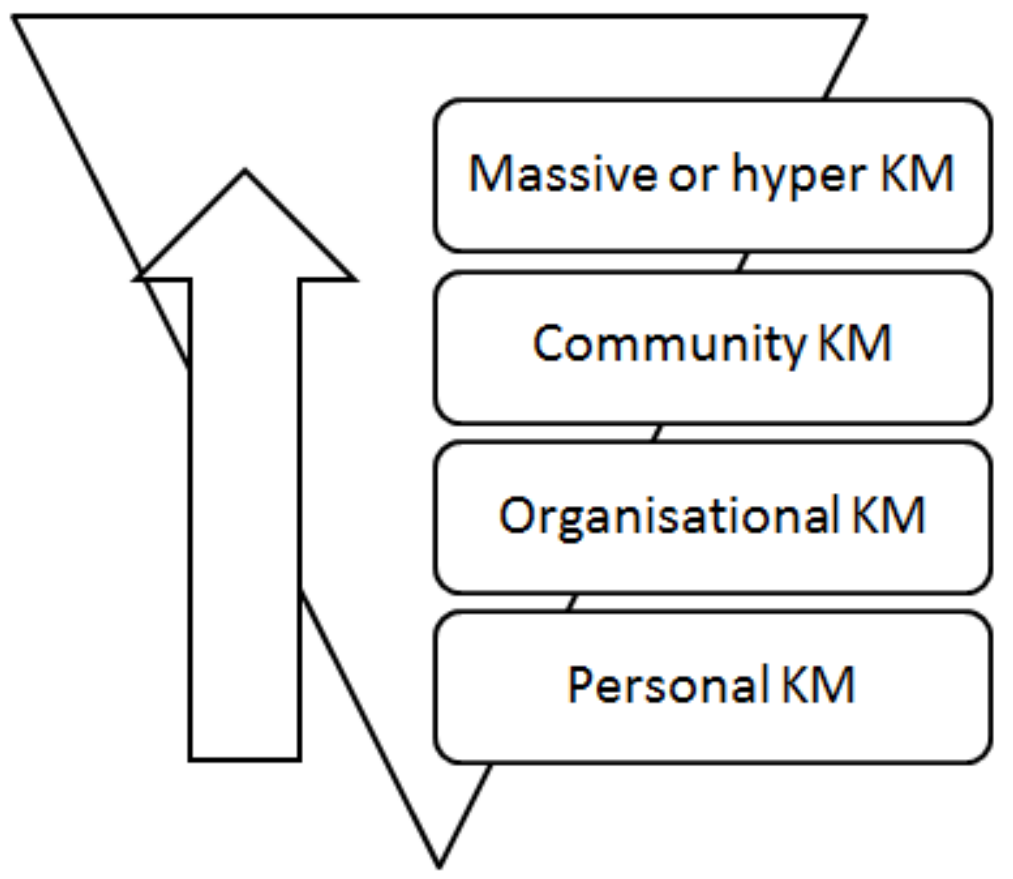

Fig 1. Les différentes phases du KM

C'est le stade de l'hyperdocumentation où ce qui n'a pas encore été exprimé le devient :

L'évolution de la Documentation se développe en six étapes. Au premier stade, l'Homme voit la Réalité de l'Univers par ses propres sens. Connaissance immédiate, intuitive, spontanée et irréfléchie. Au deuxième stade, il raisonne la Réalité et cumulant son expérience la généralisant, l'interprétant, il s'en fait une nouvelle représentation. Au troisième stade, il introduit le Document qui enregistre ce que ses sens ont perçu et ce qu'a construit sa pensée. Au quatrième stade, il crée l'instrument scientifique et la Réalité paraît alors grandie, détaillée, précisée, un autre Univers décèle successivement toutes ses dimensions. Au cinquième stade, le Document intervient à nouveau et c'est pour enregistrer directement la perception procurée par les instruments. Documents et instruments sont alors à ce point associés qu'il n'y a plus deux choses distinctes, mais une seule: le Document-Instrument. Au sixième stade, un stade de plus et tous les sens ayant donné lieu à un développement propre, une instrumentation enregistreuse ayant été établie pour chacun, de nouveaux sens étant sortis de l'homogénéité primitive et s'étant spécifiés, tandis que l'esprit perfectionne sa conception, s'entrevoit dans ces conditions l'Hyper-Intelligence. «Sens-Perception-Document» sont choses, notions soudées. Les documents visuels et 
les documents sonores se complètent d'autres documents, les tactiles, les gustatifs, les odorants et d'autres encore. À ce stade aussi l' "insensible ", l'imperceptible, deviendront sensible et perceptible par l'intermédiaire concret de l'instrumentdocument. L'irrationnel à son tour, tout ce qui est intransmissible et fut négligé, et qui à cause de cela se révolte et se soulève comme il advient en ces jours, l'irrationnel trouvera son «expression » par des voies encore insoupçonnées. Et ce sera vraiment alors le stade de l'Hyper-Documentation. (Otlet, 1934, p.429)

La vision de Paul Otlet demeure encore aujourd'hui impressionnante car on constate que la transmission des connaissances est encore en développement au niveau de l'utilisation des cinq sens notamment. L'enjeu n'est donc pas seulement celui du processus classique de gestion des connaissances via une transformation documentaire de la connaissance intuitive, mais il s'agit d'une acquisition autant par le lire que par le faire.

Les chercheurs en SHS ne sont donc pas seulement des penseurs, mais aussi des «faiseurs » (Berra, 2012). Par conséquent, l'ingénierie des connaissances requises est aussi une ingénierie des savoir-faire qui s'observent notamment avec les sites web et les carnets de recherche qui expliquent les méthodologies pour utiliser des logiciels de traitement de données. Réflexion et production sont donc mêlées. Dès lors, certains y voient un nouvel humanisme (Doueihi, $2011^{\circ}$ ) porteur d'une diversité de méthodes et de productions de connaissances qui s'observent également par la capacité à utiliser de nouveaux outils de production et de partage de connaissances. Le rapprochement avec l'idéal encyclopédique devient plus évident car l'objectif de l'encyclopédie avec ses planches détaillées supposait non seulement que le lecteur éclairé comprenne, mais qu'il soit incité à faire, refaire et innover comme l'a bien mis en avant Gilbert Simondon (Simondon, 1989. De ce fait, les humanités digitales vont produire de plus en plus des aspects méthodologiques et théoriques notamment au niveau de la masse des outils disponibles, mais également les conditions possibles de l'interprétation des nouvelles productions générées comme les cartographies, les visualisations de données ${ }^{20}$, etc. L'élargissement du modèle de la gestion des connaissances en quatre étapes montre que l'économie des connaissances qui s'ensuit implique de mieux identifier les différents acteurs et intermédiaires qui participent à son expansion. L'échelon organisationnel principal, reconnaissable notamment par la signature du chercheur sur laquelle se base les logiques de classement de type Shanghai devient nettement insuffisant, tant la mesure de la production mérite d'être étudiée différemment en prenant davantage appui sur les logiques individuelles d'évolution de carrière, mais aussi sur les groupes et communautés scientifiques institutionnels et informels qui participent à la circulation des savoirs. Mais c'est également les acteurs industriels et notamment ceux du web comme Google (nous songeons non seulement au moteur de recherche, mais également à Google Scholar ainsi qu'à Google Drive) dont il faudrait davantage étudier le rôle dans cette économie. Si des recommandations institutionnelles incitent parfois à la prudence, la première tentative d'hébergement des données en ligne de grande ampleur vient d'être seulement développée fin 2015 pour les acteurs du CNRS avec un service en ligne développé sur la solution owncloud. Ce retard

\footnotetext{
${ }^{20}$ Dans notre enquête, $37,74 \%$ déclarent avoir déjà utilisé le logiciel Gephi qui permet notamment de réaliser des cartographies et des graphes.
} 
institutionnel a laissé le champ libre depuis longtemps à des tentatives de valorisation économique par des acteurs privés. Or, c'est bien l'ensemble des étapes du processus (du PKM au MKM) qui mériterait une évaluation.

\section{Conclusion : mise à jour, collaboration et concurrence}

Les chercheurs et acteurs des humanités digitales sont présents à toutes les phases de la gestion des connaissances, si ce n'est que le second échelon plus classique dans la conception initiale de la gestion des connaissances (ce que nous avons désigné par OKM) tend à devenir d'une importance moindre par rapport aux autres échelons, et ce d'autant que la richesse d'information disponible est plus grande en dehors de l'institution locale, tout comme la reconnaissance qu'il est possible d'obtenir. La connaissance de l'autre et donc la reconnaissance par l'autre sont clairement essentielles dans les logiques de transmission de par la motivation qu'elles génèrent. Cependant, la logique concurrentielle qui existe dans les milieux de la recherche constitue une difficulté dans les démarches de partage et de solidarité. En effet, comment imaginer un système d'échanges quand il existe un phénomène d'incertitudes quant à la progression de carrière ? Dans un subtil mélange de collaboration et de concurrence (Menger, 2014), le chercheur en humanités digitales cherche à ménager ses diverses connaissances (intellectuelles comme humaines). Si l'objectif est l'amélioration de la connaissance, elle ne peut se réaliser que par des investissements personnels notables. La mise à disposition de données, de productions, d'articles, de méthodes et de méthodologies permet au chercheur de gagner en visibilité et notoriété. C'est le moteur le plus classique de la collaboration, principe méritocratique qu'on retrouve dans les communautés open source, mais aussi dans les logiques initiales de l'Internet notamment depuis la création des recommandations $\left(\mathrm{RFC}^{\circ}\right)$ par Jon Postel. Le savoir repose sur des logiques de don, il faut donc que le chercheur en Humanités digitales donne à voir, s'il veut être lui-même visible afin d'obtenir un poste. Mais la métamorphose produite par les humanités digitales va probablement aller encore plus loin tant les évolutions en organisation des connaissances finissent fréquemment par produire des métamorphoses organisationnelles (Gluschko, 2013) et institutionnelles plus profondes, ce qui fait dire à Alan Liu (Liu, 2009 ${ }^{\circ}$ ) qu'elles agissent comme un alien qui colonise peu à peu tous les espaces. Il reste à attendre quelques années encore pour mesurer les résultats de cette colonisation.

\section{Bibliographie}

BALSAMO, A. (2011), "Technohumanism and the rise of digital humanities" Aspray, William (dir.) Digital Media: Technological and Social Challenges of the Interactive World. Scarecrow Press, p.213-224.

BERRA, A. (2012), Faire des humanités numériques. OpenEdition Press. <http://books.openedition.org/oep/238>

BERRY, D. M, CITTON, Y, MASURE, A., (2015). «subjectivités computationnelles », Multitudes, $\mathrm{n}^{\circ}$ 59, p. 196-205

BERRY, D. M. (2012), Understanding Digital Humanities, Palgrave Macmillan. 
BEGUIN-VERBRUGGE, A. (2008), «Entre modélisation et représentation : le knowledge management, un défi pour les SIC ». Volant, C. (dir.), L'information dans les organisations : dynamique et complexité, Presses universitaires François-Rabelais. http://books.openedition.org/pufr/705

BOUCHEZ, J.-P. (2004), Les nouveaux travailleurs du savoir, Paris: Editions d'Organisation. BOUSTANY, J., BROUDOUX, E., CHARTRON, G. (2014). La Mediation Numerique: renouvellement et Diversification des Pratiques, De Boeck.

CITTON, Y. (2015), «Humanités numériques: une médiapolitique des savoirs encore à inventer. », Multitudes n59, p. 169-180

BOYD D., CRAWFORD, K., (2011), "Six Provocations for Big Data "; A Decade in Internet

Time: Symposium on the Dynamics of the Internet and Society, $<$ http://dx.doi.org/10.2139/ssrn.1926431>

CLIVAZ, C.,VINCK, D., (2014). «Introduction », Les Cahiers du numérique, Vol. 10(3), p. 9-16.

DOMBROWSKI, Q. (2014), What Ever Happened to Project Bamboo? Literary and Linguistic Computing, <http://doi.org/10.1093/1lc/fqu026>

DAVIDSON, C., (2010); "Humanities and Technology in the Information Age" in Frodeman, Robert, Julie Thompson Klein, and Carl Mitcham, (dir.). The Oxford Handbook of Interdisciplinarity. Oxford University Press, USA.

DEEGAN, M., McCarty W., (2012), Collaborative Research in the Digital Humanities. Ashgate Publishing Limited.

DONDIS, D. A. (1974), A Primer of Visual Literacy, MIT Press.

DOUEIHI, M., (2011) ; Pour un humanisme numérique ; Paris, Seuil.

FERRARIS, M., (2012), Documentality: Why It Is Necessary to Leave Traces, New York: Fordham University Press.

GALLEZOT, G., Le Deuff O., (2009), «Chercheurs 2.0 ?», Schöpfel J. (dir.) Communication scientifique : Les nouveaux enjeux. Les Cahiers du Numérique, vol. $5, \mathrm{n}^{\circ}$ $2,15-31$.

GLUSCHKO, R. J. (2013), The Discipline of Organizing, Cambridge, MA: MIT Press.

GUITTON, J. (1951); Le travail intellectuel, conseils à ceux qui étudient et à ceux qui écrivent, Aubier, Editions montaigne.

HAYLES, N. K., (2012), How We Think: Digital Media and Contemporary Technogenesis, , University of Chicago Press.

LE DEUFF, O. (2014), «Translittératie et transmédia », Les Cahiers du numérique 3/2014 (Vol. 10), p. 55-72

LE DEUFF , O., (2012), » Le réseau personnel de gestion des connaissances et la redéfinition du travail », Terminal $n^{\circ} 110$, p.39-54

LITTLE, G., (2011), "We Are All Digital Humanists Now”,The Journal of Academic Librarianship, Volume 37, Issue 4, July 2011, 352-354.

LIU, A., (2004a), The Laws of Cool: Knowledge Work and the Culture of Information.: The University of Chicago Press.

LIU, A., (2004b), "Transcendental Data: Toward a Cultural History and Aesthetics of the New Encoded Discourse”, Critical Inquiry, Vol. 31, No. 1 (Autumn 2004), 49-84.

Liu A., (2009), "Digital Humanities and Academic Change" "English Language Notes", 47, (Spring-Summer 2009), Spring-Summer 2009, 17-35.

MANOVICH, L., (2013), Software Takes Command, Bloomsbury Academic.

MENGER, P.-M., (2014), La différence, la concurrence et la disproportion: Sociologie du travail créateur, Fayard.

MORETTI, F., (2013); Distant Reading, Verso Books. 
OTLET, P, (1934), Traité de documentation: le livre sur le livre, théorie et pratique, Bruxelles, Editions Mundaneum, 1934,

OWWISKIE, B., (2013). "Skunks in the library : a path to production for scholarly R\&D », Journal of library administration. Digital Humanities in libraries : new models for scholarly engagement, 2013, t. 53, $\mathrm{n}^{\circ} 1$, p. 53-66.

ORY, P.,SIRINELLI,J.F. (2004). Les intellectuels en France. Paris: Tempus Perrin.

POLLARD, D., (2008) PKM: A bottom-up approach to knowledge management. In Knowledge Management in Practice: Connections and Context, ed. T.K. Srikantaiah and M.E.D. Koenig, Information Today, pp. 95-114

POUYLLAU, S., (2014), «Les digital humanities ont-elles existé ? », Le temps des humanités digitales, Olivier LE DEUFF (dir.), Fyp éditions, Limoges, p.103-114

POSNER, M., (2013). « No half measures : overcoming common challenges to doing Digital Humanities in the library », Journal of library administration. Digital Humanities in libraries : new models for scholarly engagement, 2013, t. 53, $\mathrm{n}^{\circ} 1, \mathrm{p} .43-52$.

PRESNER, T., (2010). Digital Humanities 2.0: A report of knowledge. <http://www.hastac.org/files/legacy/DigHum2.0-Presner.pdf>

READINGS, B., (1996), The university in ruins. Cambridge, Harvard University Press

SIMONDON, G., (1958), Du mode d'existence des objets techniques. Paris, Aubier.

SVENSSON, P., $\left(2010^{\circ}\right)$,“The Landscape of Digital Humanities ”. Digital Humanities. Vol. $4: 1$. Digital Humanities, 2010. <http://digitalhumanities.org/dhq/vol/4/1/000080/000080.html. $\geq$

THELY, N., SERRES, A., LE DEUFF, O, (2014), «Le THATCamp comme nouvelle forme de communication scientifique?», THATCamp Saint-Malo 2013: Non actes de la non conférence, Éditions de la Maison des sciences de l'homme.

WENGER, E., (1998), Communities of Practice: Learning, Meaning, and Identity, ,Cambridge University Press. 